\title{
Hard exclusive reactions and generalized parton distributions
}

\author{
Avetik Hayrapetyan $^{1, a}$, I. Brodski ${ }^{1}$, M. Dueren ${ }^{1}$, E. Etzelmueller ${ }^{1}$, M. Stahl ${ }^{1}$, and H. Stenzel ${ }^{1}$, \\ on behalf of the HERMES Collaboration ${ }^{\text {b }}$ \\ ${ }^{1}$ Justus-Liebig University, Giessen, Germany
}

\begin{abstract}
The recently developed formalism of Generalized Parton Distributions (GPDs) allows connecting the experimental information of hard exclusive reactions to the spin contribution and even to the angular momentum contribution of quarks in the nucleon. By selecting different quantum numbers of the final state in exclusive productions, different GPDs can be addressed separately. The HERMES experiment at the HERA ring at DESY (Hamburg) made pioneering contributions and first constraints to Generalized Parton Distributions (GPDs), using hard exclusive vector meson production (EVMP) and Deeply Virtual Compton Scattering (DVCS). Using a novel recoil detector, HERMES managed to measure DVCS and EVMP free of any significant background. Selected results are highlighted and discussed in this paper.
\end{abstract}

\section{Introduction}

The discovery of O. Stern and W. Gerlach [1], "Raumquantizierung der magnetischen Momente in Atomen", and the introduction of spin in quantum mechanics happened approximately hundred years ago. Although the formalism of spin with all its consequences and new properties is well integrated in theory, we are far away from fully understanding the origin of spin in the case of composite relativistic systems like the nucleon. Using polarized Deep Inelastic Scattering (DIS) experiments the helicity dependent Parton Density Functions (PDFs) have been measured and falsified naive expectations of the spin composition of the proton. At the end of 90's it became clear that the PDFs alone can not describe the complex composition of the nucleon spin, as especially quark and gluon angular momentum effects, which seem to have a significant contribution to the nucleon spin, are not accessible this way. The recently developed formalism of Generalized Parton Distributions (GPDs) [2] allows to parameterize the experimental information from various exclusive reactions measurements and can describe the spin contribution and even the angular momentum of partons [3] in the nucleon. GPDs actually contain the combined information of PDFs and of form factors (FFs), which are accessible in elastic scattering experiments. The GPD formalism allows to formulate a complete 3-dimensional picture of the nucleon.

Hard exclusive processes can be used to investigate GPDs. By selecting final state particles with different quantum numbers in exclusive production [4], different GPDs can be addressed separately. The most clean way from theory point of view is the deeply virtual Compton scattering (DVCS).

\footnotetext{
ae-mail: Avetik.Hayrapetyan@uni-giessen.de

${ }^{b}$ http://hermes.desy.de/
} 


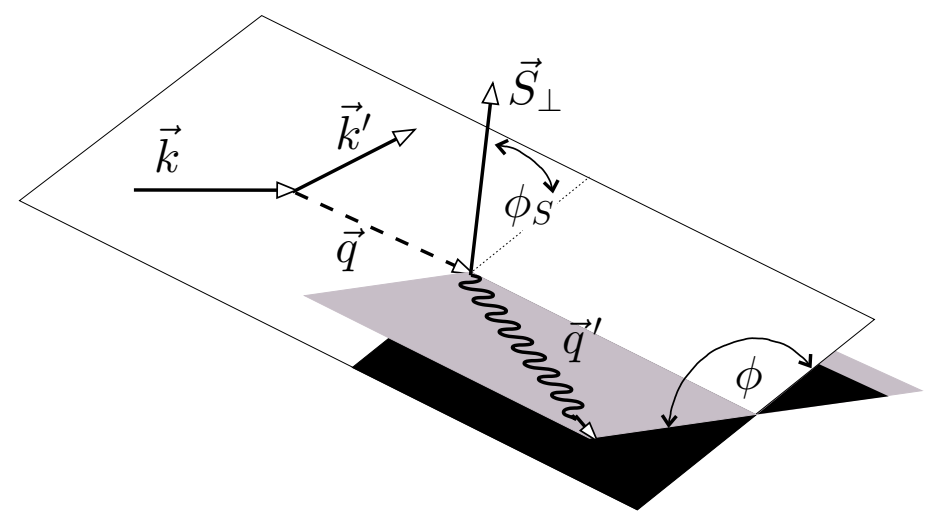

Figure 1. Momenta and azimuthal angles for exclusive real-photon electro-production in the target rest frame. The quantity $\phi$ denotes the angle between the lepton scattering plane containing the three-momenta $\mathrm{k}$ and $\mathrm{k}$ ' of the incoming and outgoing lepton and the photon production plane correspondingly defined by the vector $\mathrm{q}=\mathrm{k}-\mathrm{k}$ ' and the momentum q' of the real photon. The symbol $\phi_{S}$ denotes the angle between the lepton scattering plane and $S_{\perp}$, the component of the target polarization vector that is orthogonal to $\mathrm{q}$.

Experimentally, DVCS is hard to measure because of the normal Bremsstrahlung process (BetheHeitler) has the identical final state and a much higher cross-section at HERMES energies. However, the interference term of Bethe-Heitler and DVCS can be measured precisely, as the Bremsstrahlung process does not create single spin azimuthal asymmetries, whereas the interference term does. In addition the interference term is magnified by the amplitude of the BH term. In Fig. 1 the definition of the azimuthal angles is depicted. The cross-section depends on these angles and different GPD models predict different amplitudes for the harmonic Fourier decomposition of these angular distributions. By measuring these amplitudes in the experiment one gains constrains of different GPD models.

In this short note we summarize a few selected DVCS measurements from HERMES.

\section{The HERMES experiment}

The HERMES experiment and its spectrometer are described in detail elsewhere [5]. DVCS events were identified by detecting the scattered lepton in coincidence with the produced $\gamma$ : To guarantee the exclusivity of the events, cuts on the respective mass windows and constraints on the variable 
Table 1. Correspondence of asymmetry amplitude to be measured with the GPD function to be constrained.

\begin{tabular}{llllllll}
\hline$A_{C}^{\cos (0 \phi)}$ & $A_{C}^{\cos (\phi)}$ & $A_{C}^{\cos (2 \phi)}$ & $A_{L U}^{\sin (\phi)}$ & $A_{L U}^{\sin (2 \phi)}$ & $A_{L U, I}^{\sin (\phi)}$ & $A_{L U, I}^{\sin (2 \phi)}$ & $A_{U T, I}^{\sin \left(\phi-\phi_{s}\right)}$ \\
\hline $\operatorname{Re}(\mathrm{H})$ & $\operatorname{Re}(\mathrm{H})$ & $\operatorname{Re}(\mathrm{H})$ & $\operatorname{Im}(\mathrm{H})$ & $\operatorname{Im}(\mathrm{H})$ & $\operatorname{Im}(\mathrm{H})$ & $\operatorname{Im}(\mathrm{H})$ & $\operatorname{Im}(\mathrm{H}-\mathrm{E})$ \\
\hline
\end{tabular}

\begin{tabular}{llllllll}
\hline$A_{U T, D V C S}^{\sin \left(\phi-\phi_{s}\right)}$ & $A_{U T, I}^{\sin \left(\phi-\phi_{s}\right) \cos (\phi)}$ & $A_{U T, I}^{\sin \left(\phi-\phi_{s}\right) \sin (\phi)}$ & $A_{U L}^{\sin (\phi)}$ & $A_{U L}^{\sin (2 \phi)}$ & $A_{L L}^{\cos (0 \phi)}$ & $A_{L L}^{\cos (\phi)}$ & $A_{L L}^{\cos (2 \phi)}$ \\
\hline$E * E$ & $\operatorname{Im}(\mathrm{H}-\mathrm{E})$ & $\operatorname{Im}(\mathrm{H}-\mathrm{E})$ & $\operatorname{Im}(\tilde{H})$ & $\operatorname{Im}(\tilde{H})$ & $\operatorname{Im}(\tilde{H})$ & $\operatorname{Re}(\tilde{E})$ & $\operatorname{Re}(\tilde{E})$ \\
\hline
\end{tabular}

$$
\Delta E=\frac{M_{X}^{2}-M^{2}}{2 M}
$$

are applied, where $M_{X}$ is the missing mass and $M$ is the proton mass.

\subsection{The HERMES Recoil detector}

After first measurements of DVCS asymmetries [6] with the HERMES spectrometer HERMES decided to upgrade the detector to a system that is able to measure and reconstruct the complete event. For this purpose an additional solenoid based spectrometer was designed and constructed[7] to detect the recoil protons. This way due to the over-determination of the event kinematics a very pure measurement was feasible. The Fig. 2 illustrates the results of the above mentioned hardware development. An almost $100 \%$ cleanliness of the reaction is achieved, however with the price of a significant loss of statistics (right plot versus middle one).

\section{Results}

The HERMES new measurement indicates [8] that asymmetry amplitudes for a clean measurement might differ from first measurement [9] using only forward spectrometer. This change in terms of cleanliness is clear visible when one compares the left plot in Fig 2 with the right one. In terms of asymmetry amplitudes this comparison is depicted in Fig 3 where this change, although small in size, but is systematically visible, especially on the overall point, where the whole statistical power of measurement is accumulated. The new measurement was confronted against existing GPD models and this is shown in Fig. 4 where two models $[10,11]$ with different parameterizations are used. The Fig. 5 shows all measurements of all DVCS amplitudes measured at HERMES, averaged over all kinematic variables and shown as one average asymmetry amplitude. The correspondence of the asymmetry amplitudes and the GPD functions that are constrained by these amplitudes is given in Table 1.The full list of HERMES publications about DVCS plus associated DVCS and Exclusive Vector Meson Production and the data which have been used to constrain GPDs [12], can be found on the HERMES web site [13] .

\section{Acknowledgment}

The authors acknowledge financial support from the German funding agency BMBF and EU HP3GPDex program, grant Nr. 62300226. 


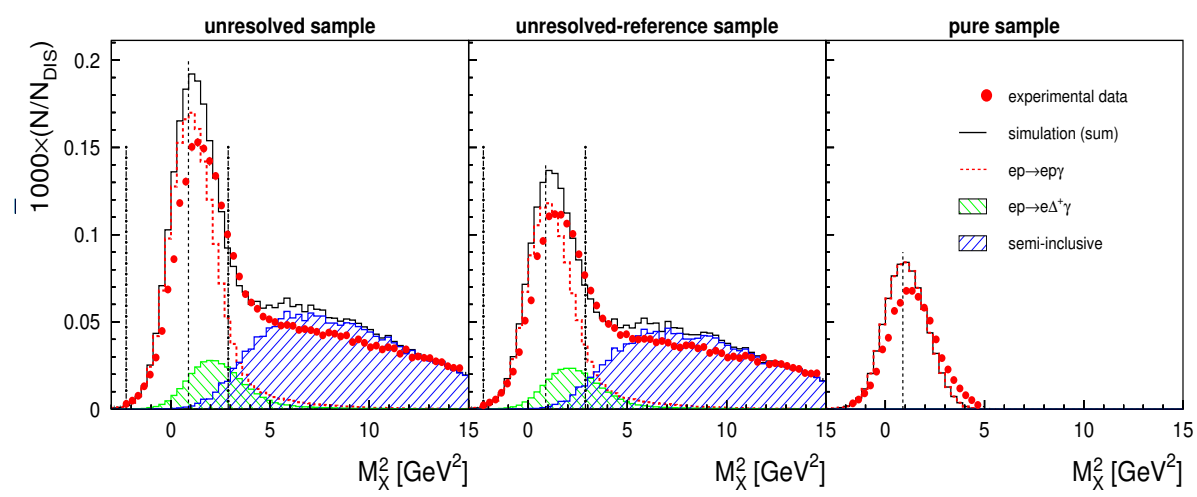

Figure 2. $M_{x}^{2}$ distribution in DVCS samples. Left: unresolved (i.e. measured only with forward spectrometer); middle: unresolved-reference (i.e. measured with only forward spectrometer, but then required that recoil proton flies into recoil detector acceptance); right: pure sample (full detection of reaction inclusive recoil proton). Experimental data, shown as data points (uncertainties covered by symbols) are compared to simulated data. In every panel, the contribution from BH events is indicated as dashed histogram, and the contributions from associated production and semi-inclusive background are shown as hatched histograms. The sum of the simulated distributions is shown as solid histogram.

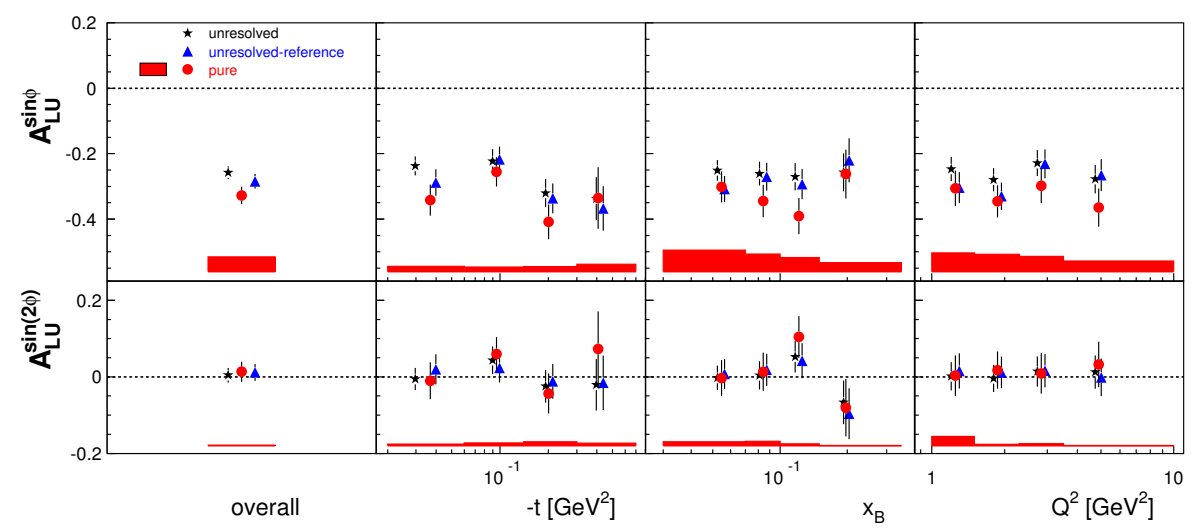

Figure 3. Amplitudes of the single-charge beam-helicity asymmetry in deeply virtual Compton scattering shown in projections of $-t, x_{\mathrm{B}}$, and $Q^{2}$. The "overall" results shown in the very left panel are extracted in a single kinematic bin covering the entire kinematic acceptance. The statistical uncertainties are presented by the error bars, while the systematic uncertainties are represented by the bands. Shown are amplitudes extracted from a pure $e p \rightarrow e p \gamma$ sample (red squares shown at their kinematic values) obtained with recoil-proton reconstruction; amplitudes extracted from an unresolved-reference sample (blue triangles shifted to the right for better visibility) without recoil-proton reconstruction but requiring its four-momentum to be in the recoil-detector acceptance; amplitudes extracted from an unresolved sample without requirements from recoil-detector acceptance and reconstruction (green circles, shifted to the left for better visibility). The latter two sets of amplitudes are subject to an average contribution of $13 \%$ for associated processes. All three sets of amplitudes are extracted from the same 2006/2007 positron-beam data set and the results are strongly statistically correlated. 


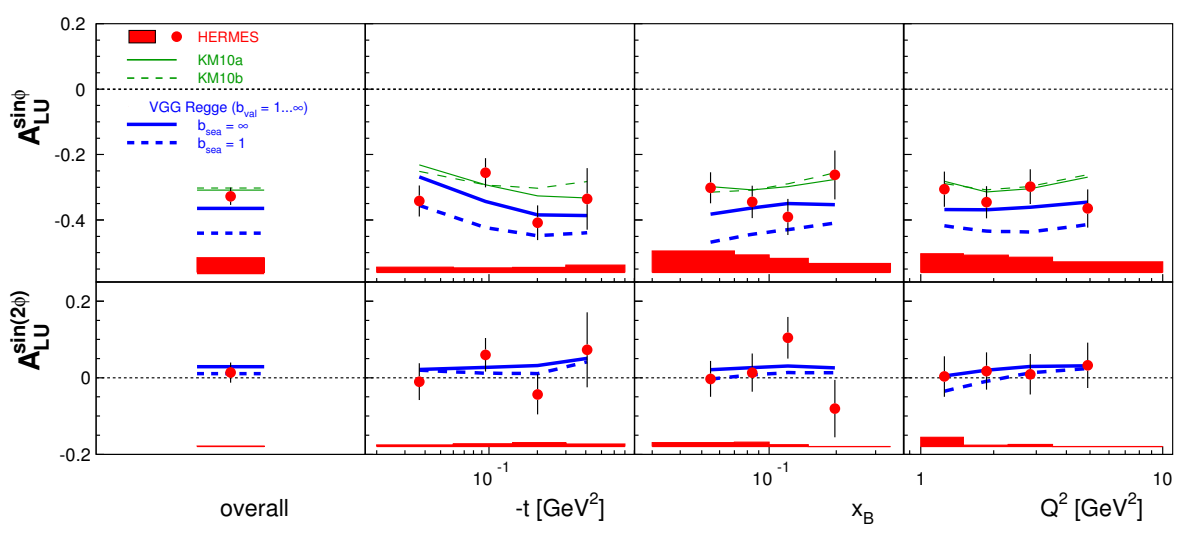

Figure 4. Amplitudes of single-charge beam-helicity asymmetry extracted from pure sample obtained with recoil-proton reconstruction. The amplitudes are presented in projections of $-t, x_{B}$, and $Q^{2}$. The overall results shown in the very left panel are extracted in a single kinematic bin covering the entire kinematic acceptance. Statistical (systematic) uncertainties are represented by error bars (bands). A separate scale uncertainty arising from the measurement of the beam polarization amounts to $1.96 \%$. The theoretical models are evaluated at the average values of the kinematics. The thickness of the VGG lines represents the range bval $=1 \ldots \infty$.

\section{References}

[1] W. Gerlach and O. Stern, Z. Phys. 8 (1922) 110.

[2] D. Muller at al., Fortsch. Phys. 42 (1994) 101;

A.V. Radushkin, Phys. Rev. D 56 (1997) 5524.

[3] X. Ji, J. Phys. G 24 (1998) 1191.

[4] K. Goeke et al., Prog. Part. Nucl. Phys. 41 (2001) 401.

[5] HERMES Collaboration, K Ackerstaff et al., Nucl. Inst. and Meth. A417(1998) 230.

[6] "Measurement of the beam spin azimuthal asymmetry associated with deeply virtual Compton scattering", HERMES Collaboration, A. Airapetian et al., Phys.Rev.Lett. 87 (2001) 182001.

[7] The HERMES Recoil Detector, A. Airapetian et al., JINST 8 (2013) P05012.

[8] A. Airapetian et al., JHEP10(2012)042.

[9] A. Airapetian et al.,JHEP 07 (2012) 032.

[10] M. Vanderhaegen et al., Phys. Rev. D 68 (2003).

[11] K. Kumericki and D. Mueller, Nucl. Phys. B 81 (2010), 1.

[12] M. Guidal, EPJ Web Conf. 73 (2014) 01007.

[13] http://www-hermes.desy.de/notes/pub/publications.html 


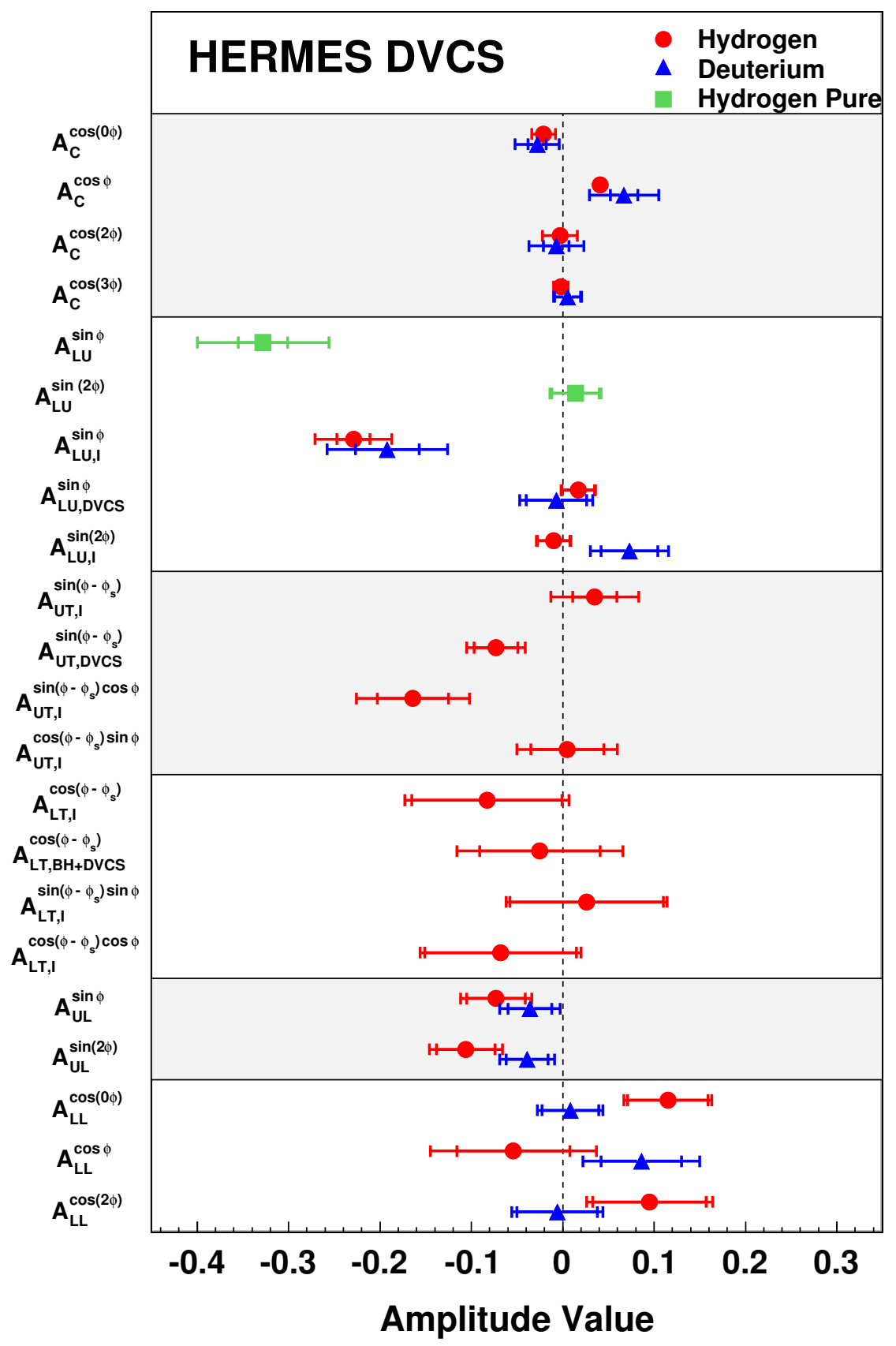

Figure 5. DVCS overview plot showing all azimuthal asymmetry amplitudes in the entire HERMES kinematic range ("overall"/"integrated") from data taken in the years 1996-2007. This includes data on the unpolarized hydrogen and deuterium targets, the longitudinally polarized hydrogen and deuterium targets and the transversely polarized hydrogen target. Shown are the leading amplitudes of the beam charge, the beam helicity, the target spin and the double spin asymmetries, which are all sensitive to different GPDs (real or imaginary part of corresponding Compton form factors). The inner error bars give the statistical uncertainties, the outer error bars the quadratic sum of statistical and systematic uncertainties. Also shown measurement done with Recoil detector, named as Hydrogen pure. 